\section{Excesso de peso em adultos do Estado de Pernambuco, Brasil: magnitude e fatores associados}

\author{
Overweight among adults in Pernambuco State, \\ Brazil: prevalence and associated factors
}

\footnotetext{
1 Universidade Federal de Pernambuco, Recife, Brasil. 2 Secretaria de Desenvolvimento Sociale Direitos Humanos, Recife Brasil.

3 Instituto de Medicina Integral Professor Fernando Figueira, Recife, Brasil.

Correspondência C. P. S. Pinho Universidade Federal de Pernambuco.

Rua Bianor de Oliveira 262, Recife, PE 52040-350, Brasil. claudiasabinopinho@hotmail. com
}

\begin{abstract}
In order to evaluate the prevalence of overweight and associated factors in Pernambuco State, Brazil, a cross-sectional population-based study was conducted in 2006, including 1,580 adults 25 to 59 years of age. Overweight was defined as body mass index $\geq 25 \mathrm{~kg} / \mathrm{m} 2$. The conceptual model included demographic, socioeconomic, reproductive, and behavioral variables. Prevalence ofoverweight was 51.1\% (95\%CI: 48.6-53.6) and was statistically associated with age over 40 years $(P R=$ 1.27; 95\%CI: 1.10-1.46), female gender ( $P R=$ 1.29; 95\%CI: 1.16-1.43), former smoking ( $P R=$ 1.42; 95\%CI: 1.20-1.69), higher income ( $P R=$ 1.49; 95\%CI: 1.30-1.71), and history of early pregnancy ( $<18$ years) $(P R=1.25$; 95\%CI: 1.11-1.66). There was no association with alcohol consumption, level of physical activity, or specific foods. The high prevalence of overweight is consistent with epidemic levels of this problem elsewhere in world, and the association with several factors supports its multifactor etiology.
\end{abstract}

Body Mass Index; Overweight; Epidemiologic Factors; Adult

\author{
Claudia Porto Sabino Pinho ${ }^{1}$ \\ Alcides da Silva Diniz 1 \\ Ilma Kruze Grande Arruda 1 \\ Pedro Israel Cabral de Lira 1 \\ Leopoldina Augusta de Souza Sequeira 1 \\ Fabiana Cristina Lima da Silva Pastich \\ Gonçalves ${ }^{2}$ \\ Malaquias Batista Filho ${ }^{3}$
}

\section{Introdução}

O excesso de peso, definido pelo índice de massa corpórea (IMC) $\geq 25 \mathrm{~kg} / \mathrm{m}^{2}$, está relacionado ao aumento da morbimortalidade por predispor o indivíduo a graves transtornos na pressão arterial e no metabolismo dos lipídios e glicídios, tendo como consequências mais marcantes doenças cardio e cerebrovasculares, principais causas de morte e incapacitação de adultos em todo o mundo 1,2 .

A prevalência mundial de sobrepeso e obesidade vem apresentando um rápido e progressivo aumento nas últimas décadas, sendo caracterizada como uma verdadeira epidemia mundial 3 . Resultados da Pesquisa de Orçamentos Familiares (POF), realizada no Brasil em 2008-2009 4, revelam que o excesso de peso atingiu cerca de metade dos homens $(50,1 \%)$ e das mulheres (48\%).

Dados da II Pesquisa Estadual de Saúde e Nutrição 5, realizada em 1997, com amostra representativa do Estado de Pernambuco, revelou prevalência de excesso de peso no sexo masculino e feminino de $34,2 \%$ e $43 \%$, respectivamente. Em Recife, dados provenientes de estudo realizado por inquérito telefônico, em 2006, revelam que o excesso de peso esteve presente em $43,3 \%$ da população 6 .

O IMC é o indicador de obesidade total mais utilizado em estudos epidemiológicos 7,8, sendo um bom parâmetro para expressar a gordura cor- 
poral em excesso e útil para quantificar a obesidade global. Apesar de suas limitações (variações na estrutura e proporções corporais, além de pontos de corte único para ampla faixa etária), o IMC é a medida mais aceita universalmente para categorizar o estado nutricional dos indivíduos, sobretudo, em escala populacional 9.

Diversos fatores biológicos, socioeconômicos e comportamentais, associados a uma predisposição genética, têm sido associados ao excesso de peso e à obesidade. Entretanto, devido à sua etiologia multifatorial é difícil mensurar a força de cada uma das variáveis envolvidas no processo do ganho excessivo de peso 10 .

O Estado de Pernambuco não dispõe de dados atualizados acerca da magnitude do excesso de peso e dos fatores que estão influenciando o perfil antropométrico de sua população. Além disso, estudos oriundos de amostras representativas, englobando população de áreas rurais e urbanas que enfoquem os possíveis fatores determinantes do excesso de peso corporal, ainda são escassos na Região Nordeste do país. Nesse contexto, o presente estudo teve como objetivo descrever a magnitude do excesso de peso e seus fatores associados na população adulta do Estado de Pernambuco.

\section{Métodos}

Foi realizado estudo de corte transversal, de base populacional, no período de maio a outubro de 2006, envolvendo adultos de ambos os sexos, na faixa etária de 25 a 59 anos, provenientes de área urbana e rural do Estado de Pernambuco. Foram excluídos os indivíduos que apresentaram alguma limitação física que impossibilitasse a aferição das medidas antropométricas e as gestantes.

Para definição do tamanho amostral foi considerada uma prevalência de excesso de peso estimada em $40 \%$, um erro de estimação de até $4 \%$, um nível de $95 \%$ de confiança, e um efeito do desenho da ordem de 2,5, totalizando 1.440 indivíduos a serem estudados. Para permitir um melhor nível de estratificação das variáveis independentes, o tamanho amostral foi aumentado em $25 \%$, resultando em uma amostra final de 1.800 adultos.

O processo de seleção da amostra (probabilística e estratificada) desenvolveu-se em três estágios: (1) sorteio dos municípios; (2) sorteio dos setores censitários (unidades territoriais demarcadas pelo Instituto Brasileiro de Geografia e Estatística - IBGE); (3) sorteio aleatório dos domicílios dentro de cada setor censitário para selecionar as famílias seguindo-se a incorporação de outras famílias por ordem contrária ao movimento dos ponteiros de relógio até completar a quota amostral prevista para cada setor (40 \pm 5 famílias).

A partir do sorteio aleatório, foram selecionados 18 municípios e considerados três estratos geográficos: Região Metropolitana do Recife, Interior Urbano e Interior Rural. Para definição da quantidade de setores censitários a serem estudados, foi considerado o número com representações proporcionais à população dos municípios selecionados figurando 16 setores censitários da Região Metropolitana do Recife, 17 do Interior Urbano e 12 do Interior Rural.

A aplicação dos questionários de pesquisa e a coleta das medidas antropométricas foram realizadas por duplas de técnicos treinados. A altura foi determinada com estadiômetro portátil (Alturaexata Ltda., São Paulo, Brasil), com precisão de até $1 \mathrm{~mm}$ em toda a sua extensão. Os indivíduos foram colocados em posição ereta, descalços, com membros superiores pendentes ao longo do corpo, os calcanhares, o dorso e a cabeça tocando a coluna de madeira. O peso dos adultos foi obtido utilizando-se balança digital (modelo MEA-03200, Plenna, São Paulo, Brasil), com capacidade de $150 \mathrm{~kg}$ e escala de $100 \mathrm{~g}$, com o indivíduo descalço e indumentária mínima.

Para garantir a acurácia das mensurações foram aferidas duas medidas de peso e altura e quando a diferença entre as avaliações excedia $0,5 \mathrm{~cm}$ para altura e $100 \mathrm{~g}$ para o peso, repetia-se a mensuração e anotavam-se as duas medições com valores mais próximos, sendo utilizada a média destas.

$\mathrm{O}$ excesso de peso foi considerado quando o IMC $\geq 25 \mathrm{~kg} / \mathrm{m}^{2}$, segundo ponto de corte preconizado pela Organização Mundial da Saúde (OMS) 11 .

O modelo hipotético causal construído para explicar o excesso de peso considerou variáveis demográficas: sexo, idade (categorizada nos intervalos 25|-30 anos; 30|-40 anos; 40|-50 anos e 50|-60 anos), área geográfica da residência (Região Metropolitana do Recife, Interior Urbano e Interior Rural); socioeconômicas: escolaridade, em anos completos de estudo (categorizada nos intervalos: 0-4 anos, 5-8 anos e $\geq 9$ anos), e renda familiar em salário mínimo per capita (estabelecida em quartis de renda, e levando-se em conta o salário mínimo vigente na época: $\mathrm{R} \$ 350,00$ ); reprodutivas: paridade (categorizada em 0-3 gestações e $\geq 3$ gestações), idade da menarca (categorizada nos intervalos: $\leq 11$ anos; 12-13 anos e $\geq$ 14 anos), idade da primeira gestação $(<18$ anos e $\geq 18$ anos) e uso de contraceptivo oral (com a resposta dicotômica sim ou não); e comportamentais: tabagismo, consumo de álcool, atividade física e consumo alimentar. 
Para o tabagismo foram consideradas as categorias: fumante (o indivíduo que referiu o hábito de fumar), não fumante (o indivíduo que relatou nunca haver fumado) e ex-fumante (o indivíduo que referiu o hábito de fumar em algum momento da vida, mas que não o fazia na ocasião da aplicação do questionário). Para a variável consumo de álcool, avaliou-se o consumo de bebidas alcoólicas nos 30 dias anteriores à aplicação do questionário, sendo considerada a resposta dicotômica sim ou não.

Para determinação do nível de atividade física da população deste estudo foi utilizado o International Physical Activity Questionnaire (IPAQ; http://www.ipaq.ki.se/ipaq, acessado em 10/Set/2010), em sua versão curta, que leva em consideração as quatro dimensões da atividade física: no lazer, atividades domésticas, atividades ocupacionais e atividades relacionadas ao deslocamento. Esse instrumento mede a frequência e duração das atividades físicas moderadas, vigorosas e caminhadas realizadas na última semana por pelo menos dez minutos contínuos, incluindo exercício, esportes, atividades físicas ocupacionais e recreacionais realizadas em casa, no tempo livre, como meio de transporte e no lazer.

Um escore de atividade física em minutos por semana foi construído, somando-se os minutos despendidos em caminhada e atividades de intensidade moderada com os minutos despendidos em atividades de intensidade vigorosa multiplicados por dois. Esta estratégia visa considerar as diferentes intensidades de cada atividade, e está de acordo com as recomendações atuais quanto à prática de atividade física 12 . Um escore abaixo de 150 minutos por semana foi o ponto de corte utilizado para classificar os indivíduos como insuficientemente ativos ou sedentários 13 .

As informações referentes ao consumo alimentar foram coletadas utilizando um questionário de frequência alimentar e a avaliação do consumo foi realizada com base na metodologia proposta por Fornés et al. 14, na qual o cômputo geral da frequência de consumo é convertido em escores. Foram constituídos três grupos de alimentos: o grupo 1 foi composto por alimentos-fonte de fibras, como leguminosas, frutas, legumes e hortaliças, considerados protetores para as doenças cardiovasculares e para o ganho excessivo de peso; o grupo 2 foi composto pelos alimentos-fonte de carboidratos simples (bolo, biscoito, açúcar e refrigerantes); e no grupo 3 foram inseridos os alimentos-fonte de gorduras saturadas (carnes com gordura, frango com pele, vísceras, embutidos, laticínios, gorduras e frituras). Os grupos 2 e 3 foram compostos por alimentos considerados de risco para o desen- volvimento de doenças cardiovasculares e para o ganho de peso excessivo. Os escores de consumo de cada grupo foram categorizados em quartis.

O protocolo do estudo foi aprovado pelo Comitê de Ética em Pesquisa em Seres Humanos do Instituto de Medicina Integral Professor Fernando Figueira (IMIP), sob o número 709/2006. Os indivíduos que concordaram em participar da pesquisa assinaram um termo de consentimento livre e esclarecido.

A base de dados foi compilada no programa Epi Info versão 6.04 (Centers for Disease Control and Prevention, Atlanta, Estados Unidos), com dupla entrada, e posterior uso do módulo validate para checar eventuais erros de digitação. Para as análises estatísticas, foi empregado o SPSS versão 12.0 (SPSS Inc., Chicago, Estados Unidos).

A análise estatística foi realizada em três etapas. Inicialmente, fez-se uma análise descritiva para caracterizar a distribuição da ocorrência dos eventos, incluindo a frequência de cada variável do estudo. Na segunda etapa, realizou-se uma análise univariada estratificada por sexo entre o desfecho (excesso de peso) e as variáveis independentes para determinação da razão de prevalência (RP) e seu respectivo intervalo de 95\% de confiança (IC95\%). Numa terceira etapa, foi realizada análise multivariada, utilizando o programa Stata/SE 9.0 (Stata Corp., College Station, Estados Unidos), pelo método de regressão log-Poisson onde foram incluídas no modelo múltiplo todas as variáveis associadas ao evento de interesse com significância estatística de até 20\%. Para aceitação das associações investigadas no modelo final, foi adotado o valor de $\mathrm{p}<0,05$.

\section{Resultados}

Dos 1.800 indivíduos avaliados, ocorreram 10\% de perdas (ausência de respostas e inconsistências de informações) e 2,2\% foram excluídos por serem gestantes, totalizando 1.580 adultos efetivamente estudados.

Foi observada uma mediana de idade de 33 anos (IQ $=29-42$ ), sendo $58 \%$ do sexo feminino, $48,7 \%$ residentes do interior rural do estado e $54,3 \%$ com baixa escolaridade (menos de 5 anos completos de estudo). A mediana da renda familiar per capita (em salários mínimos) foi de 0,25 (IQ = 0,12-0,45).

Quanto às variáveis comportamentais, observou-se predominância dos indivíduos não fumantes $(62,5 \%)$, com nível suficiente de atividade física $(71,5 \%)$ e que consumiram bebidas alcoólicas nos 30 dias anteriores a realização da pesquisa $(68,9 \%)$. As medianas do escore de consumo dos grupos de alimentos fontes em fibras, 
carboidratos simples e gorduras saturadas foram de 2,33 (IQ = 1,67-3,17), 4,20 (IQ = 2,47-4,53) e 3,50 (IQ = 2,47-4,53), respectivamente.

Foi observada prevalência de excesso de peso de $51,1 \%$ (IC95\%: 48,6-53,6), e o baixo peso foi constatado em menos de $3 \%$ da população (Tabela 1).

A análise univariada para o sexo masculino (Tabela 2) mostrou uma menor suscetibilidade do EP em homens do interior rural, de menor escolaridade e renda.

Entre as mulheres, observaram-se maior prevalência do excesso de peso na menor faixa de escolaridade e um aumento pari passu com a progressão da idade (Tabela 3).

A prevalência de excesso de peso foi maior em homens e mulheres ex-fumantes quando comparada ao grupo de indivíduos fumantes e não fumantes. Não houve associação entre o excesso de peso e as outras variáveis comportamentais (consumo de álcool, nível de atividade física e consumo alimentar) (Tabelas 2 e 3 ).

Dentre as variáveis reprodutivas, apenas a idade da primeira gestação foi associada ao desfecho estudado, sendo a gestação precoce (idade $<18$ anos) um fator de risco para o excesso de peso.

$\mathrm{Na}$ análise ajustada para o sexo masculino, verificou-se entre os homens uma associação do excesso de peso com o estrato geográfico da residência, com a renda e com o tabagismo ( $\mathrm{Ta}-$ bela 4).

Entre as mulheres, apenas a idade atual e a idade da primeira gestação mantiveram-se associadas ao excesso de peso, após controle das variáveis de confusão, sendo evidenciadas maiores prevalências de excesso de peso entre mulheres de maior idade e entre aquelas em que a primeira gestação ocorreu antes dos 18 anos (Tabela 4).

\section{Discussão}

A literatura especializada 15,16 tem indicado diferentes razões para o surgimento e a manutenção da obesidade em diferentes populações. Os inquéritos que têm sido empreendidos correlacionando aspectos genéticos à ocorrência de obesidade não têm sido capazes de evidenciar a possível interferência em mais de um quarto dos obesos, fazendo com que se acredite que o processo de acúmulo excessivo de gordura corporal, na maioria dos casos, seja desencadeado por aspectos socioambientais 15,17.

Sabe-se que a grande limitação do delineamento transversal é a dificuldade de estabelecer causalidade uma vez que, tratando-se de um corte no tempo, os possíveis determinantes e o desfecho são vistos em um mesmo momento, impossibilitando a utilização da temporalidade como critério causal.

Em nossa casuística foram incluídos indivíduos apenas a partir dos 25 anos, o que poderia comprometer a representatividade da população adulta em seu conjunto. Esse é um fato conjuntural justificado pela circunstância de que os dados primários deste estudo são provenientes de pesquisa desenhada para avaliação de doenças crônicas, optando-se, em virtude da limitação de recursos, pela não inclusão dos indivíduos mais jovens (20-24 anos).

A elevada prevalência de excesso de peso $(51,1 \%)$ e a reduzida prevalência de baixo peso $(2,7 \%)$, observadas entre os adultos pernambucanos seriam um retrato do processo de transição nutricional experimentado pelo Brasil nas últimas décadas.

O percentual expressivo de indivíduos com excesso de peso foi similar aos dados apresentados pela POF 2008-2009 4, que identificaram que

Tabela 1

Estado nutricional, segundo o índice de massa corporal (IMC) de adultos de ambos os sexos, de 25 a 59 anos. Estado de Pernambuco, Brasil, 2006

\begin{tabular}{|c|c|c|c|c|c|c|c|c|c|}
\hline \multirow[t]{2}{*}{ Estado nutricional (IMC) } & \multicolumn{3}{|c|}{$\begin{array}{c}\text { Total } \\
(\mathrm{n}=1.580)\end{array}$} & \multicolumn{3}{|c|}{$\begin{array}{l}\text { Sexo masculino } \\
\qquad(n=664)\end{array}$} & \multicolumn{3}{|c|}{$\begin{array}{l}\text { Sexo feminino } \\
\quad(n=916)\end{array}$} \\
\hline & $\mathbf{n}$ & $\%$ & IC95\% & $\mathbf{n}$ & $\%$ & IC95\% & $\mathbf{n}$ & $\%$ & IC95\% \\
\hline Baixo peso $\left(<18,5 \mathrm{~kg} / \mathrm{m}^{2}\right)$ & 42 & 2,7 & $1,92-3,58$ & 11 & 1,7 & $0,8-2,9$ & 31 & 3,4 & $2,3-4,8$ \\
\hline Eutrofia $\left(18,5-24,9 \mathrm{~kg} / \mathrm{m}^{2}\right)$ & 731 & 46,3 & $43,8-48,4$ & 362 & 54,5 & $50,6-58,3$ & 369 & 40,3 & $37,1-43,5$ \\
\hline Sobrepeso $\left(25-29,9 \mathrm{~kg} / \mathrm{m}^{2}\right)$ & 532 & 33,7 & $31,3-36,1$ & 206 & 31,0 & $27,5-34,7$ & 326 & 35,6 & $32,4-38,8$ \\
\hline Obesidade $\left(\geq 30 \mathrm{~kg} / \mathrm{m}^{2}\right)$ & 275 & 17,4 & $15,6-19,4$ & 85 & 12,8 & $10,3-15,6$ & 190 & 20,7 & $18,1-23,5$ \\
\hline
\end{tabular}

IC95\%: intervalo de 95\% de confiança. 
Razão de prevalência (RP) do excesso de peso (IMC $\geq 25 \mathrm{~kg} / \mathrm{m}^{2}$ ) em homens segundo características demográficas, socioeconômicas, reprodutivas e comportamentais de adultos de 25 a 59 anos. Estado de Pernambuco, Brasil, 2006.

\begin{tabular}{|c|c|c|c|c|c|}
\hline \multirow[t]{2}{*}{ Variáveis } & \multicolumn{2}{|c|}{$\mathrm{IMC} \geq 25 \mathrm{~kg} / \mathrm{m}^{2}$} & \multirow[t]{2}{*}{$\mathrm{RP}$} & \multirow[t]{2}{*}{ IC95\% } & \multirow[t]{2}{*}{ Valor de $\mathrm{p}$} \\
\hline & $\mathrm{n}$ & $\%$ & & & \\
\hline Idade (anos) & & & & & 0,519 \\
\hline $25 \mid-30$ & 72 & 41,6 & 1,00 & - & \\
\hline $30 \mid-40$ & 129 & 44,8 & 1,08 & $0,87-1,34$ & \\
\hline $40 \mid-50$ & 49 & 40,5 & 0,97 & $0,74-1,29$ & \\
\hline $50 \mid-60$ & 41 & 50,0 & 1,20 & $0,91-1,59$ & \\
\hline Área de residência & & & & & $<0,001$ \\
\hline Região Metropolitana do Recife & 84 & 51,6 & 1,00 & - & \\
\hline Interior urbano & 86 & 56,6 & 1,10 & $0,90-1,35$ & \\
\hline Interior rural & 121 & 34,7 & 0,67 & $0,55-0,83$ & \\
\hline \multicolumn{6}{|l|}{ Escolaridade (anos de estudo) } \\
\hline $0-4$ & 137 & 36,5 & 0,62 & $0,51-0,75$ & $<0,001$ \\
\hline $5-8$ & 59 & 46,8 & 0,79 & $0,79-1,00$ & \\
\hline$\geq 9$ & 88 & 59,1 & 1,00 & - & \\
\hline Renda familiar per capita (salários mínimos) & & & & & $<0,001$ \\
\hline$\leq 0,12$ & 52 & 29,2 & 1,00 & - & \\
\hline $0,12-0,25$ & 70 & 40,9 & 1,40 & $1,05-1,87$ & \\
\hline $0,25-0,45$ & 62 & 42,2 & 1,44 & $1,07-1,94$ & \\
\hline$\geq 0,45$ & 103 & 64,0 & 2,19 & $1,69-2,83$ & \\
\hline Tabagismo & & & & & 0,001 \\
\hline Fumante & 60 & 32,6 & 1,00 & - & \\
\hline Não fumante & 166 & 47,6 & 1,46 & $1,15-1,85$ & \\
\hline Ex-fumante & 65 & 49,6 & 1,52 & $1,16-1,99$ & \\
\hline Consumo de álcool nos últimos 30 dias & & & & & 0,212 \\
\hline Não & 124 & 40,4 & 1,00 & - & \\
\hline Sim & 134 & 45,4 & 1,12 & $0,93-1,35$ & \\
\hline Atividade física * & & & & & 0,069 \\
\hline Suficientemente ativo & 222 & 42,0 & 1,00 & - & \\
\hline Insuficientemente ativo & 69 & 50,7 & 1,21 & $0,99-1,46$ & \\
\hline \multicolumn{6}{|l|}{ Consumo alimentar ** } \\
\hline \multicolumn{6}{|l|}{ Escore - Grupo 1 (quartis) } \\
\hline$\leq 1,67$ & 67 & 41,9 & 0,94 & $0,73-1,20$ & 0,522 \\
\hline $1,67-2,33$ & 77 & 48,4 & 1,08 & $0,86-1,36$ & \\
\hline $2,33-3,17$ & 70 & 40,9 & 0,91 & $0,72-1,17$ & \\
\hline$\geq 3,17$ & 77 & 44,8 & 1,00 & - & \\
\hline \multicolumn{6}{|l|}{ Escore - Grupo 2 (quartis) } \\
\hline$\leq 3,03$ & 68 & 40,2 & 1,00 & - & 0,308 \\
\hline $3,03-4,20$ & 80 & 49,4 & 1,23 & $0,96-1,56$ & \\
\hline $4,20-5,33$ & 71 & 45,5 & 1,13 & $0,88-1,45$ & \\
\hline$\geq 5,33$ & 72 & 41,1 & 1,11 & $0,86-1,42$ & \\
\hline Escore - Grupo 3 (quartis) & & & & & 0,146 \\
\hline$\leq 2,47$ & 65 & 37,8 & 1,00 & - & \\
\hline $2,47-3,50$ & 71 & 43,3 & 1,15 & $0,88-1,49$ & \\
\hline $3,50-4,53$ & 80 & 50,3 & 1,33 & $1,04-1,70$ & \\
\hline$\geq 4,53$ & 73 & 45,1 & 1,19 & $0,92-1,54$ & \\
\hline
\end{tabular}

IC95\%: intervalo de 95\% de confiança; IMC: índice de massa corporal.

* Suficientemente ativos: atividades moderada, vigorosa ou caminhada por tempo $\geq 150$ minutos/semana. Insuficientemente ativos ou sedentários: indivíduos com atividades moderada, vigorosa ou caminhada < 150 minutos/semana;

** Grupo 1: Frutas, legumes, hortaliças e leguminosas; Grupo 2: Bolo, biscoito, açúcar e refrigerante; Grupo 3: carnes com gordura, frango com pele, vísceras, embutidos, leite e derivados, gorduras e frituras. 
Tabela 3

Razão de prevalência (RP) do excesso de peso (IMC $\geq 25 \mathrm{~kg} / \mathrm{m}^{2}$ ) em mulheres segundo características demográficas, socioeconômicas, reprodutivas e comportamentais de adultos de 25 a 59 anos. Estado de Pernambuco, Brasil, 2006.

\begin{tabular}{|c|c|c|c|c|c|}
\hline \multirow[t]{2}{*}{ Variáveis } & \multicolumn{2}{|c|}{$\geq 25 \mathrm{~kg} / \mathrm{m}^{2}$} & \multirow[t]{2}{*}{ RP } & \multirow[t]{2}{*}{ IC95\% } & \multirow[t]{2}{*}{ Valor de $p$} \\
\hline & $\mathbf{N}$ & $\%$ & & & \\
\hline Idade (anos) & & & & & $<0,001$ \\
\hline $25 \mid-30$ & 145 & 46,5 & 1,00 & - & \\
\hline $30 \mid-40$ & 188 & 54,5 & 1,17 & $1,01-1,37$ & \\
\hline $40 \mid-50$ & 115 & 68,9 & 1,46 & $1,25-1,71$ & \\
\hline $50 \mid-60$ & 68 & 75,6 & 1,63 & $1,38-1,92$ & \\
\hline Área de residência & & & & & 0,696 \\
\hline Região Metropolitana do Recife & 141 & 54,4 & 1,00 & - & \\
\hline Interior urbano & 138 & 58,2 & 1,07 & $0,92-1,25$ & \\
\hline Interior rural & 237 & 56,4 & 1,04 & $0,90-1,19$ & \\
\hline \multicolumn{6}{|l|}{ Escolaridade (anos de estudo) } \\
\hline $0-4$ & 288 & 60,8 & 1,29 & $1,10-1,51$ & 0,01 \\
\hline $5-8$ & 124 & 55,6 & 1,17 & $0,98-1,41$ & \\
\hline$\geq 9$ & 102 & 47,2 & 1,00 & - & \\
\hline Renda familiar per capita (salários mínimos) & & & & & 0,247 \\
\hline$\leq 0,12$ & 113 & 52,6 & 1,00 & - & \\
\hline $0,12-0,25$ & 132 & 54,8 & 1,04 & $0,88-1,24$ & \\
\hline $0,25-0,45$ & 125 & 56,8 & 1,08 & $0,91-1,28$ & \\
\hline$\geq 0,45$ & 140 & 61,7 & 1,17 & $1,00-1,38$ & \\
\hline Tabagismo & & & & & 0,006 \\
\hline Fumante & 85 & 50,3 & 1,00 & - & \\
\hline Não fumante & 355 & 55,6 & 1,11 & $0,94-1,30$ & \\
\hline Ex-fumante & 75 & 69,4 & 1,38 & $1,14-1,68$ & \\
\hline Consumo de álcool nos últimos 30 dias & & & & & 0,71 \\
\hline Não & 90 & 54,9 & 1,00 & - & \\
\hline Sim & 401 & 56,5 & 1,03 & $0,88-1,20$ & \\
\hline Atividade física * & & & & & 0,165 \\
\hline Suficientemente ativo & 349 & 58,0 & 1,00 & - & \\
\hline Insuficientemente ativo & 167 & 53,2 & 1,09 & $0,96-1,23$ & \\
\hline \multicolumn{6}{|l|}{ Consumo alimentar ** } \\
\hline \multicolumn{6}{|l|}{ Escore - Grupo 1 (quartis) } \\
\hline$\leq 1,67$ & 130 & 55,3 & 0,98 & $0,83-1,16$ & 0,961 \\
\hline $1,67-2,33$ & 122 & 57,8 & 1,03 & $0,87-1,21$ & \\
\hline $2,33-3,17$ & 139 & 56,0 & 0,99 & $0,85-1,17$ & \\
\hline$\geq 3,17$ & 124 & 56,4 & 1,00 & - & \\
\hline \multicolumn{6}{|l|}{ Escore - Grupo 2 (quartis) } \\
\hline$\leq 3,03$ & 131 & 60,6 & 1,00 & - & 0,314 \\
\hline $3,03-4,20$ & 142 & 56,6 & 0,93 & $0,80-1,09$ & \\
\hline $4,20-5,33$ & 117 & 51,8 & 0,85 & $0,72-1,01$ & \\
\hline$\geq 5,33$ & 125 & 56,3 & 0,93 & $0,79-1,09$ & \\
\hline Escore - Grupo 3 (quartis) & & & & & 0,092 \\
\hline$\leq 2,47$ & 139 & 60,7 & 1,00 & - & \\
\hline $2,47-3,50$ & 136 & 59,6 & 0,98 & $0,85-1,14$ & \\
\hline $3,50-4,53$ & 112 & 50,2 & 0,83 & $0,70-0,98$ & \\
\hline$\geq 4,53$ & 128 & 54,7 & 0,90 & $0,77-1,05$ & \\
\hline
\end{tabular}

(continua) 
Tabela 3 (continuação)

\begin{tabular}{|c|c|c|c|c|c|}
\hline \multirow[t]{2}{*}{ Variáveis } & \multicolumn{2}{|c|}{$\geq 25 \mathrm{~kg} / \mathrm{m}^{2}$} & \multirow[t]{2}{*}{$\mathrm{RP}$} & \multirow[t]{2}{*}{ IC95\% } & \multirow[t]{2}{*}{ Valor de $p$} \\
\hline & $\mathbf{N}$ & $\%$ & & & \\
\hline Paridade (gestações) & & & & & 0,113 \\
\hline $0-3$ & 223 & 53,7 & 1,00 & - & \\
\hline$\geq 3$ & 289 & 59,0 & 1,10 & $0,98-1,23$ & \\
\hline \multicolumn{6}{|c|}{ Idade da menarca (anos) } \\
\hline$\leq 11$ & 73 & 61,3 & 1,00 & - & 0,109 \\
\hline $12-13$ & 202 & 54,4 & 0,89 & $0,75-1,05$ & \\
\hline$\geq 14$ & 158 & 52,3 & 0,85 & $0,71-1,02$ & \\
\hline \multicolumn{6}{|c|}{ Idade da 1a gestação (anos) } \\
\hline$<18$ & 134 & 64,1 & 1,00 & - & 0,004 \\
\hline$\geq 18$ & 300 & 51,2 & 0,80 & $0,70-0,91$ & \\
\hline \multicolumn{6}{|c|}{ Uso de contraceptivo oral } \\
\hline Não & 326 & 55,0 & 1,00 & - & 0,616 \\
\hline Sim & 112 & 52,3 & 0,95 & $0,82-1,10$ & \\
\hline
\end{tabular}

IC95\%: intervalo de 95\% de confiança; IMC: índice de massa corporal.

* Suficientemente ativos: atividades moderada, vigorosa ou caminhada por tempo $\geq 150$ minutos/semana. Insuficientemente ativos ou sedentários: indivíduos com atividades moderada, vigorosa ou caminhada < 150 minutos/semana;

** Grupo 1: frutas, legumes, hortaliças e leguminosas; Grupo 2: bolo, biscoito, açúcar e refrigerante; Grupo 3: carnes com gordura, frango com pele, vísceras, embutidos, leite e derivados, gorduras e frituras.

cerca de metade da população adulta brasileira tinha excesso de peso. Outros estudos de base populacional realizados no Norte e Nordeste do país 18,19,20 revelaram prevalências menores, enquanto avaliações realizadas em populações do Sul e Sudeste indicaram prevalências similares aos nossos achados 3,21,22, demonstrando que o Estado de Pernambuco apresenta padrão de excesso de peso similar às regiões mais desenvolvidas do país.

Dados da II Pesquisa Estadual de Saúde e Nutrição, realizada em 19975 no Estado de Pernambuco, com metodologia similar e considerando os mesmos municípios analisados neste estudo, mostraram prevalência de excesso de peso em $39,6 \%$ dos adultos. Indicando, portanto, que houve um crescimento de cerca de $30 \%$ do excesso de peso entre pernambucanos com idade acima dos 25 anos em um período inferior a 10 anos. Outros estudos brasileiros mostram claras tendências regionais e temporais do aumento progressivo da obesidade no país 23,24 .

A maior prevalência do excesso de peso observada entre as mulheres investigadas nesse estudo poderia ser atribuída a alguns fatores, tais como maior composição de gordura no gênero, gestações, diferenças hormonais e climatério 25,26 . Esse achado é consistente com os resultados descritos em outros estudos populacionais que avaliaram os fatores associados ao excesso de peso e obesidade em mulheres 16,18,26,27. En- tretanto, apesar de uma forte tendência apontar que a obesidade abdominal é mais prevalente entre as mulheres, o papel do sexo em relação ao excesso de peso e obesidade global não está bem definido, sendo discordantes os resultados que analisam essa associação. Inquérito telefônico que avaliou os fatores de risco para doenças crônicas nas 26 capitais brasileiras e no Distrito Federal indicou que o excesso de peso tendeu a ser mais frequente em homens do que em mulheres, exceto nas cidades de Recife, Rio Branco, Salvador e São Paulo, onde as frequências foram semelhantes nos dois sexos 6 . Trata-se, provavelmente, de uma característica recente da transição nutricional no Brasil.

A associação positiva entre a idade e o excesso de peso poderia ser explicada, em parte, pelo declínio da taxa metabólica basal que fisiologicamente acompanha o processo de envelhecimento e à redução natural da prática de atividades físicas ${ }^{28}$. Esse resultado é consistente com a literatura que aponta relação direta entre o aumento do peso e a idade do indivíduo $3,16,26,27,29$. No entanto, essa constatação foi limitada ao sexo feminino. Resultados registrados pela POF 2002-2003 29 indicam comportamento diferenciado do excesso de peso entre os sexos, ou seja, enquanto na mulher há uma tendência crescente do excesso de peso à proporção que aumenta a idade, entre os homens há um declínio a partir dos 55 anos. 
Modelo final (regressão de Poisson) dos fatores associados ao excesso de peso (IMC $\geq 25 \mathrm{~kg} / \mathrm{m}^{2}$ ), em homens e mulheres na faixa etária de 25 a 59 anos. Estado de Pernambuco, Brasil, 2006.

\begin{tabular}{|c|c|c|c|}
\hline Variáveis & $\mathrm{RP}$ & IC95\% & Valor de $p$ \\
\hline \multicolumn{4}{|l|}{ Homens } \\
\hline \multicolumn{4}{|l|}{ Área de residência } \\
\hline Região Metropolitana do Recife & 1,00 & - & 0,014 \\
\hline Interior urbano & 1,19 & $0,97-1,47$ & \\
\hline Interior rural & 0,88 & $0,70-1,11$ & \\
\hline Renda familiar em salários mínimos * per capita (quartis) & & & $<0,001$ \\
\hline$\leq 0,12$ & 1,00 & - & \\
\hline $0,12-0,25$ & 1,32 & $0,99-1,77$ & \\
\hline $0,25-0,45$ & 1,29 & $0,95-1,74$ & \\
\hline$\geq 0,45$ & 1,91 & $1,44-2,53$ & \\
\hline Tabagismo & & & 0,012 \\
\hline Fumante & 1,00 & - & \\
\hline Não fumante & 1,37 & $1,08-1,72$ & \\
\hline Ex-fumante & 1,10 & $1,12-1,91$ & \\
\hline \multicolumn{4}{|l|}{ Mulheres } \\
\hline Idade (anos) & & & $<0,001$ \\
\hline $25 \mid-30$ & 1,00 & - & \\
\hline $30 \mid-40$ & 1,19 & $1,02-1,39$ & \\
\hline $40 \mid-50$ & 1,51 & $1,31-1,73$ & \\
\hline $50 \mid-60 * \star$ & - & - & \\
\hline Idade da 1ạ gestação (anos) & & & $<0,001$ \\
\hline$<18$ & 1,00 & - & \\
\hline$\geq 18$ & 0,83 & $0,69-0,89$ & \\
\hline
\end{tabular}

IC95\%: intervalo de 95\% de confiança; IMC: índice de massa corporal; RP: razão de prevalência.

* Valor do salário mínimo no período: $\mathrm{R} \$ 350,00$;

** Esta categoria de idade não entrou no modelo ajustado porque apresentou forte colinearidade com a variável dependente.

É provável que a menor prevalência de excesso de peso encontrada nos homens provenientes de área rural esteja relacionada a fatores alimentares e ao nível de atividade física. Considerando se tratar de área rural, pressupõe-se um maior gasto energético, representado pelo tipo de trabalho desenvolvido e pelos deslocamentos percorridos, além de um menor acesso a alimentos industrializados, que comumente têm elevada densidade calórica. Essa combinação beneficiaria o homem rural no processo de ganho excessivo de peso.

A associação direta entre o excesso de peso e a renda observada entre os homens, mesmo após ajuste das variáveis de confusão, corrobora resultados encontrados por outros autores 20,27, indicando uma relação positiva entre o excesso de peso e a renda familiar apenas no sexo masculino.
A tendência inversa nos sexos na análise da associação entre o excesso de peso e a escolaridade é algo que parece estar se desenhando na análise do acúmulo de gordura corporal. Portanto, a relação direta entre o excesso de peso e o nível de escolaridade entre as mulheres e a relação inversa entre os homens, uma constatação já descrita por outros autores $16,25,27$, pode representar uma nova tendência: maiores cuidados com o controle do peso corporal apenas nas mulheres em função dos níveis crescentes de educação formal.

Maior excesso de peso entre as mulheres com idade prematura ao primeiro parto poderia ser explicado pela ativação precoce dos hormônios relacionados ao ciclo reprodutivo, propiciando assim um aumento da concentração de gordura corporal 30. Na literatura ainda são escassos os estudos que avaliam a associação deste fator com a adiposidade corporal. 
A ausência de associação entre o excesso de peso e paridade foi um resultado inesperado, uma vez que a alta paridade destaca-se entre os fatores contributivos para a obesidade em mulheres. Essa tendência tem sido amplamente descrita na literatura $3,18,26,31,32$, e poderia ser explicada pelo ganho excessivo de peso no período gestacional e posterior dificuldade para perda, sobretudo nas mulheres que não amamentam segundo as recomendações normativas. Nos países em desenvolvimento, nos quais o número maior de gestações por mulher é acompanhado de um tempo menor entre as gestações, essa relação é ainda mais expressiva 32 .

A maior prevalência de excesso de peso entre os indivíduos ex-fumantes é um resultado que corrobora dados de outros estudos 16,31,33 que demonstram que os fumantes que abandonaram o hábito de fumar são os que apresentam maiores prevalências de obesidade e este poderia ser um dos maiores impedimentos para cumprir o abandono 34 . Além disso, indivíduos fumantes parecem ter uma certa proteção para o ganho excessivo de peso, atribuindo-se esta condição à ação da nicotina, que induz à supressão do apetite, provavelmente pelo aumento dos neurotransmissores anorexígenos dopamina e serotonina, intensificada pelo possível aumento sérico de leptina nos fumantes. Outra ação da nicotina é o aumento da atividade adrenérgica que induz a termogênese e a consequente redução do peso corporal 35 .

Apesar de não se ter encontrado associação do excesso de peso com o consumo de álcool, diversos estudos têm avaliado esse papel na alteração dos indicadores de obesidade global e central 16,18,31,33. Entretanto ainda não está claro o papel do álcool no mecanismo de determinação da adiposidade corporal, sobretudo pela grande variação metodológica encontrada na literatura relativa à frequência e quantidade do etanol consumido. Além disso, da mesma forma que a teratogenicidade do álcool encontra diferentes respostas dependendo de características individuais, o ganho de peso relacionado ao consumo de etanol segue a mesma tendência ${ }^{36}$. Deve-se ressaltar que a seleção metodológica para avaliação do consumo do álcool no nosso estudo (consumo nos 30 dias anteriores) é possivelmente um fator limitante para análise da associação com o excesso de peso, sobretudo porque esta definição não reflete a frequência ou quantidade de etanol consumida.

Os dois aspectos mais apresentados na literatura como determinantes de um quadro de balanço energético positivo têm sido mudanças no padrão alimentar, com aumento do fornecimento de energia pela dieta, e redução da atividade fí- sica, configurando o que poderia ser chamado de estilo de vida ocidental contemporâneo 15,18,37. Entretanto, os resultados aqui apresentados não destacaram essa associação, sendo esta condição passível de ser interpretada à luz da causalidade reversa, dado que, uma vez diagnosticado o excesso de peso corporal, o indivíduo pode ter iniciado medidas de aumento do gasto energético e mudanças no padrão alimentar para o controle do problema.

É importante destacar, com relação à atividade física, que se verificou uma baixa prevalência de indivíduos considerados sedentários ou insuficientemente ativos (28,5\%), contrastando com a expressiva prevalência de excesso de peso evidenciada em nossos resultados. Entretanto, como a mensuração da atividade física foi realizada por um instrumento que considera as atividades desenvolvidas no lazer, deslocamento, e em atividades domésticas e ocupacionais, justifica-se esse baixo percentual encontrado. Além disso, os índices de sedentarismo encontrados foram equivalentes aos obtidos em estudos recentes que utilizaram o mesmo instrumento de avaliação e mesmo ponto de corte 12,38.

A expressiva prevalência do excesso de peso revelada nesta investigação corrobora os níveis epidêmicos que este problema tem assumido em todo o mundo, incluindo as regiões do país menos desenvolvidas. Ademais, a constatação de que vários fatores foram associados ao excesso de peso, reforçam a multifatorialidade na etiologia do problema e indicam a necessidade de ações intersetoriais articuladas e estruturação de macropolíticas sociais para o controle da doença.

A ocorrência de excesso de peso em homens e mulheres parece estar sendo influenciada por fatores distintos. Portanto, prioridades de intervenção devem ser dirigidas aos subgrupos onde os problemas nutricionais foram mais frequentes: mulheres a partir dos 40 anos de idade, de menor escolaridade e com primeira gestação antes dos 18 anos, adultos ex-fumantes de ambos os sexos, homens de maior nível socioeconômico e residentes na Região Metropolitana do Recife. 


\section{Resumo}

Com o objetivo de avaliar a magnitude do excesso de peso e fatores associados em adultos do Estado de Pernambuco, Brasil, foi realizado estudo transversal, de base populacional, em 2006, envolvendo 1.580 adultos, na faixa etária de 25-59 anos. O excesso de peso foi determinado pelo índice de massa corporal $\geq 25 \mathrm{~kg} /$ $\mathrm{m}^{2}$. O modelo conceitual considerou variáveis socioeconômicas/demográficas, reprodutivas e comportamentais. A prevalência de excesso de peso foi de $51,1 \%$ (IC95\%: 48,6-53,6). A análise multivariada mostrou que o excesso de peso foi maior a partir de 40 anos $(R P=1,27$; IC95\%: 1,10-1,46), em mulheres $(R P=1,29$; IC95\%: 1,16-1,43), em ex-fumantes ( $R P=1,42$; IC95\%: 1,20-1,69), em indivíduos com maior renda ( $R P=1,49$; IC95\%: 1,30-1,71) e em mulheres com primeira gestação com idade < 18 anos (RP = 1,25; IC95\%: 1,11-1,66). Não houve associação com o consumo de álcool, com a atividade física e com o consumo alimentar. A expressiva prevalência do excesso de peso corrobora os níveis epidêmicos que este problema tem assumido em todo o mundo e a associação com vários fatores e reforçam a multifatorialidade de sua etiologia.

Índice de Massa Corporal; Sobrepeso; Fatores Epidemiológicos; Adulto

\section{Colaboradores}

C. P. S. Pinho participou da concepção do estudo, redação do artigo e análise estatística. A. S. Diniz e I. K. G. Arruda contribuíram com análise estatística e revisão da versão final. P. I. C. Lira, F. C. L. S. P. Gonçalves e M. Batista Filho participaram da concepção do estudo e revisão da versão final do artigo. L. A. S. Sequeira participou da revisão da versão final do artigo.

\section{Agradecimentos}

Ao CNPq (processos no. 505540/2004-5 e 501989/2005-4).

\section{Referências}

1. World Health Organization. Physical status: the use and interpretation of anthropometry. Geneva: World Health Organization; 1995. (WHO Technical Report Series, 854).

2. Santos RD, Timerman S, Sposito AC. Diretrizes para cardiologistas sobre excesso de peso e doença cardiovascular dos Departamentos de Aterosclerose, Cardiologia Clínica e FUNCOR da Sociedade Brasileira de Cardiologia. Arq Bras Cardiol 2002; 78:1-13.

3. Velásquez-Melendez G, Pimenta AM, Kac G. Epidemiologia do sobrepeso e da obesidade e seus fatores determinantes em Belo Horizonte (MG), Brasil: estudo transversal de base populacional. Rev Panam Salud Pública 2004; 16:308-14.

4. Instituto Brasileiro de Geografia e Estatística. Pesquisa de Orçamentos Familiares - 2008-2009: aquisição alimentar domiciliar per capita, Brasil e grandes regiões. Rio de Janeiro: Instituto Brasileiro de Geografia e Estatística; 2010.
5. Ferraciu CCS. Prevalência de sobrepeso e obesidade em adultos maiores de dezoito anos no estado de Pernambuco-1997 [Dissertação de Mestrado]. Recife: Centro de Ciências da Saúde, Universidade Federal de Pernambuco; 2005.

6. Moura EC, Morais Neto OL, Malta DC, Moura L, Silva LN, Bernal R, et al. Vigilância de fatores de risco para doenças crônicas por inquérito telefônico nas capitais do 26 estados brasileiros e no Distrito Federal (2006). Rev Bras Epidemiol 2008; 11: 20-37.

7. Guedes DP. Recursos antropométricos para análise da composição corporal. Rev Bras Educ Fís Esp 2006; 20: 115-9.

8. Abrantes MM, Lamounier JA, Colosimo AE. Prevalência de sobrepeso e obesidade nas regiões Nordeste e Sudeste do Brasil. Rev Assoc Med Bras 2003; 49:162-6.

9. Organização Mundial da Saúde. Obesidade: prevenindo e controlando a epidemia global. São Paulo: Organização Mundial da Saúde; 2000. 
10. Bernardi F, Cichelero C, Vitolo MR. Comportamento de restrição alimentar e obesidade. Rev Nutr 2005; 18:85-93.

11. World Health Organization. Obesity: preventing and managing the global epidemic. Report of a WHO Consultation on Obesity. Geneva: World Health Organization; 1998.

12. Alves JGB, Siqueira FV, Figueiroa JN, Facchini LA, Silveira DS, Piccini RX, et al. Prevalência de adultos e idosos insuficientemente ativos moradores em áreas de unidades básicas de saúde com e sem Programa Saúde da Família em Pernambuco, Brasil. Cad Saúde Pública 2010; 26:543-56.

13. US Department of Health and Human Services. Physical activity patterns in modern culture. http:// www.enotes.com/public-health-encyclopedia/ physical-activity (acessado em 15/Nov/2010).

14. Fornés NS, Martins IS, Velasquez-Melendez G, Latorre MRDO. Escores de consumo alimentar e níveis lipêmicos em população de São Paulo, Brasil. Rev Saúde Pública 2002; 36:12-8.

15. Mendonça CP, Anjos LA. Aspectos das práticas alimentares e da atividade física como determinantes do crescimento do sobrepeso/obesidade no Brasil. Cad Saúde Pública 2004; 20:698-709.

16. Gigante D, Barros F, Post C, Olinto M. Prevalência de obesidade em adultos e seus fatores de risco. Rev Saúde Pública 1997; 31:236-46.

17. Stunkard AJ. Factores determinantes de la obesidad: opinión actual. In: Peña M, Bacallao J, editores. La obesidad en la pobreza: un nuevo reto para la salud pública. Washington DC: Organización Panamericana de la Salud; 2000. p. 27-32.

18. Oliveira LPM, Assis AMO, Silva MCM, Santana MLP, Santos NS, Pinheiro SMC, et al. Fatores associados a excesso de peso e concentração de gordura abdominal em adultos na cidade de Salvador, Bahia, Brasil. Cad Saúde Pública 2009; 25:570-82.

19. Borges HP, Cruz NC, Moura EC. Associação entre hipertensão arterial e excesso de peso em adultos, Belém, Pará, 2005. Arq Bras Cardiol 2008; 91: 110-8.

20. Veloso HJF, Silva AUM. Prevalência e fatores associados à obesidade abdominal e ao excesso de peso em adultos maranhenses. Rev Bras Epidemiol 2010: 13:400-12.

21. Carvalhaes AR, Moura EC, Monteiro CA. Prevalência de fatores de risco para doenças crônicas: inquérito populacional mediante entrevistas telefônicas em Botucatu, São Paulo, 2004. Rev Bras Epidemiol 2008; 11:14-23.

22. Gigante DP, Dias-da-Costa JS, Olinto MTA, Menezes AMB, Macedo S. Obesidade em população adulta de Pelotas, Rio Grande do Sul, Brasil e associação com nível sócio-econômico. Cad Saúde Pública 2006; 22:1873-9.

23. Monteiro CA, Conde WL. A tendência secular da obesidade segundo estratos sociais: Nordeste e Sudeste do Brasil. Arq Bras Endocrinol Metabol 1999; 43:186-94.

24. Batista Filho M, Rissin A. A transição nutricional no Brasil: tendências regionais e temporais. Cad Saúde Pública 2003; 19 Suppl 1:S181-91.
25. Monteiro CA, Conde WL, Castro IRR. A tendência cambiante da relação entre escolaridade e risco de obesidade no Brasil (1975-1997). Cad Saúde Pública 2003; 19 Suppl 1:S67-75.

26. Ronsoni RM, Coutinho MSSA, Pereira MR, Silva RH, Becker IC, Sehnen Jr. L. Prevalência de obesidade e seus fatores associados na população de Tubarão-SC. ACM Arq Catarin Med 2005; 34:51-7.

27. Vedana EHB, Peres MA, Neves J, Rocha GC, Longo GZ. Prevalência de obesidade e fatores potencialmente causais em adultos em região Sul do Brasil. Arq Bras Endocrinol Metab 2008; 52:1156-62.

28. Francischi RPP, Pereira LO, Freitas MS, Klopfer M, Santos RC, Vieira O, et al. Obesidade: atualização sobre sua etiologia, morbidade e tratamento. Rev Nutr 2000; 13:17-28.

29. Instituto Brasileiro de Geografia e Estatística. Pesquisa de orçamentos familiares 2002-2003. Análise da disponibilidade domiciliar de alimentos e do estado nutricional no Brasil. Rio de Janeiro: Instituto Brasileiro de Geografia e Estatística; 2004.

30. Kac G, Velásquez-Meléndez G, Valente JG. Menarca, gravidez precoce e obesidade em mulheres brasileiras selecionadas em um Centro de Saúde de Belo Horizonte, Minas Gerais, Brasil. Cad Saúde Pública 2003; 19:111-8.

31. Oliveira EO, Velásquez-Melendez G, Kac G. Fatores demográficos e comportamentais associados à obesidade abdominal em usuárias de centro de saúde de Belo Horizonte, Minas Gerais, Brasil. Rev Nutr 2007; 20:361-9.

32. França AP, Aldrighi JM, Marucci MFN. Fatores associados à obesidade global e à obesidade abdominal em mulheres na pós-menopausa. Rev Bras Saúde Matern Infant 2008; 8:65-73.

33. Castanheira M, Olinto MTA, Gigante DP. Associação de variáveis sócio-demográficas e comportamentais com a gordura abdominal em adultos: estudo de base populacional no Sul do Brasil. Cad Saúde Pública 2003; 19 Suppl 1:S55-65.

34. Pisabarro R, Irrazábal E, Recalde A. Primera encuesta nacional de sobrepeso y obesidad (ENSO 1). Rev Méd Urug 2000; 16:31-8.

35. Berto SJP, Carvalhaes MABL, Moura EC. Tabagismo associado a outros fatores comportamentais de risco de doenças e agravos crônicos não transmissíveis. Cad Saúde Pública 2010; 26:1573-82.

36. Kachani AT, Brasiliano S. Hochgraf PB. O impacto do consumo alcoólico no ganho de peso. Rev Psiquaitr Clín (São Paulo) 2008; 35:21-4.

37. Tardido AP, Falcão MF. O impacto da modernização na transição nutricional e obesidade. Rev Bras Nutr Clín 2006; 21:117-24.

38. Hallal PC, Victora CG, Wells JC, Lima RC. Physical inactivity: prevalence and associated variables in Brazilian adults. Med Sci Sports Exerc 2003; 35:1894-900.

Recebido em 26/Mai/2011

Versão final reapresentada em 31/Ago/2011

Aprovado em 15/Set/2011 